\title{
Relação das variáveis climáticas com os casos de dengue em um município do interior de Mato Grosso dos anos 2001 a 2015
}

\section{Relationship of climate variables with the cases of dengue in a interior municipality of Mato Grosso state from the} years 2001 to 2015

Relación de las variables climáticas con los casos de dengue en un municipio del interior de Mato Grosso desde los años 2001 a 2015

Débora Aparecida da Silva Santos ${ }^{1}$ Juliana Zenaro Rodrigues ${ }^{2}$ Ricardo Alves de Olinda ${ }^{3}$ Letícia Silveira Goulart ${ }^{4}$

${ }^{1}$ Enfermeira. Doutora em Recursos Naturais pela Universidade Federal de Campina Grande (UFCG). Professora, Adjunto I, Curso de Enfermagem da Universidade Federal de Mato Grosso (UFMT), Campus Universitário de Rondonópolis (CUR), Área de Saúde Coletiva. Pesquisadora nas áreas de saúde coletiva, estudos epidemiológicos e saúde ambiental. E-mail: deboraassantos@hotmail.com

2 Enfermeira. Residente pelo Programa de Residência Multiprofissional em Saúde da Família (PREMSAF) na Universidade Federal de Mato Grosso (UFMT), Campus Universitário de Rondonópolis (CUR). E-mail: julianazenaro@hotmail.com

${ }^{3}$ Estatístico. Doutor em Estatística. Professor Adjunto da Universidade Estadual da Paraíba (UEPB), Campina Grande, PB. E-mail: ricardo.estat@yahoo.com.br

${ }^{4}$ Farmacêutica. Doutora. Professora, Associada I, Curso de Enfermagem da Universidade Federal de Mato Grosso (UFMT), Campus Universitário de Rondonópolis (CUR). E-mail: Igoulart77@yahoo.com.br 
Resumo: Este estudo analisou a relação das variáveis climáticas com casos de dengue em Rondonópolis, MT, entre 2001 e 2015. Tipo ecológico, de série temporal e analítico, abordagem quantitativa e descritiva. Dados de fonte secundária, utilizando as variáveis casos de dengue notificados e temperatura, umidade do ar e precipitação. Foram ajustados modelos de Poisson e binomial negativo, adotando-se nível de significância de 5\% $(p<0,05)$. Foram utilizados os testes te qui-quadrado e análise de resíduos. Estimou que o número de casos de dengue diminua em aproximadamente $43 \%$ a cada 1 grau centígrado de aumento da temperatura média; aumento em torno de $77 \%$ a cada unidade de aumento na temperatura máxima; e, em torno de $17 \%$, a cada $1 \%$ de aumento da umidade. Essa associação revela elevado índice de notificação de dengue nos meses quentes e úmidos, sugerindo desenvolvimento de ações interdisciplinares e intersetoriais prioritariamente para essa época do ano.

Palavras-chave: dengue; clima; atenção primária à saúde; pesquisa interdisciplinar.

\begin{abstract}
This study analyzed the relationship of climatic variables with dengue cases in Rondonópolis, MT, between 2001 and 2015. Ecological type, time and analytical series, quantitative and descriptive approach. Secondary source data using reported cases of dengue and temperature, air humidity and precipitation as variables. Poisson and negative binomial models were adjusted, adopting a significance level of $5 \%(p<0.05)$. The t-test and chi-square and residue analysis were used. It was estimated that the number of dengue cases decreases approximately $43 \%$ at each 1 centigrade degree elevated in mean temperature; increases about $77 \%$ at each enlarged unit in the maximum temperature; and increases about $17 \%$ at each $1 \%$ raised in humidity. This association reveals a high rate of notification of dengue in the warm and humid months, suggesting the development of interdisciplinary and intersectoral actions, primarily for this period of the year.
\end{abstract}

Keywords: dengue; climate; primary health care; interdisciplinary research.

Resumen: Este estudio analizó la relación de las variables climáticas con casos de dengue en Rondonópolis, MT, entre 2001 y 2015. Tipo ecológico, de serie temporal y analítico, abordaje cuantitativo y descriptivo. Datos de fuente secundaria, utilizando las variables de casos de dengue notificados y temperatura, humedad del aire y precipitación. Se ajustaron modelos de Poisson y binomial negativo, adoptando un nivel de significancia del 5\% ( $p$ $<0,05)$. Se utilizaron el test $t$ y qui-cuadrado y análisis de residuos. Estimó que el número de casos de dengue disminuye en aproximadamente $43 \%$ en cada 1 grado centígrado de aumento de la temperatura media; aumente alrededor del $77 \%$ a cada unidad de aumento en la temperatura máxima; y aumente en torno al $17 \%$ cada $1 \%$ de aumento de la humedad. Esta asociación revela un elevado índice de notificación de dengue en los meses cálidos y húmedos, sugiriendo el desarrollo de acciones interdisciplinarias e intersectoriales, prioritariamente para esta época del año.

Palabras clave: dengue; clima; atención primaria a la salud; investigación interdisciplinaria. 


\section{INTRODUÇÃO}

A dengue constitui-se um problema de saúde pública no mundo, sendo considerada a mais importante arbovirose que afeta o ser humano. É causada pelo vetor Aedes Aegypti, cuja proliferação tem relação com as variáveis climáticas, sendo o verão o período de maior transmissão. As regiões endêmicas para essa doença são os países tropicais e subtropicais, nos quais as condições do ambiente favorecem o ciclo de vida do vetor.

As variações climáticas interferem diretamente no processo saúde-doença, provocando impactos positivos e negativos na qualidade de vida e de saúde das populações. A temática saúde e meio ambiente deve ser objeto de estudo, visando à compreensão da importância de entender que o comportamento dos fatores ambientais interfere diretamente nesse processo, considerando que a saúde engloba o ambiente como um de seus determinantes.

A responsabilidade pelas questões dos impactos ambientais quantitativos e qualitativos como consequência antrópica e que influenciam na saúde é um dos diversos atores sociais, incluindo os cidadãos, o Estado, os Conselhos e as instituições não governamentais. Além disso, as ações devem ser realizadas por profissionais de equipe multidisciplinar e integrada, nos âmbitos municipal, estadual e federal, considerando o Sistema Único de Saúde (SUS) como o eixo articulador do atendimento às necessidades integralizadas, por meio da promoção, prevenção e recuperação da saúde dos indivíduos e da coletividade.

Nessa interface entre saúde e meio ambiente, as variáveis climáticas como temperatura e umidade relativa do ar e a precipitação pluviométrica devem ser estudadas e associadas às questões da saúde, incluindo a dengue. Enquanto doença infecciosa reemergente, vem apresentando aumento em sua incidência de casos notificados, denotando uma preocupação para os gestores na saúde pública.

Diante do exposto, este estudo objetivou analisar a relação das variáveis climáticas com os casos de dengue em um município do interior de Mato Grosso no período de 2001 a 2015. 


\section{METODOLOGIA}

\section{1 Área e período do estudo}

Esta pesquisa foi realizada com dados relativos ao município de Rondonópolis, localizado no Estado de Mato Grosso, MT, do período de janeiro de 2001 a dezembro de 2015, equivalente a quinze anos.

Rondonópolis possui uma população de 222.316 habitantes, tendo a área da unidade territorial equivalente a $4.686,622 \mathrm{~km}^{2}$ e densidade demográfica 47 hab/ $/ \mathrm{km}^{2}$ (IBGE, 2017). O bioma é o cerrado e apresenta clima tropical úmido. Rondonópolis faz parte da microrregião 538-Rondonópolis, que é constituída por 08 municípios, somando 288.017 habitantes. Localiza-se, geograficamente, na mesorregião sudeste do Estado de Mato Grosso, com latitude de $16^{\circ} 28^{\prime} 15^{\prime \prime}$ Sul e longitude de $54^{\circ} 38^{\prime} 08^{\prime \prime}$ Oeste (IBGE, 2017). No que tange ao clima, no Brasil, foram classificados três zonas e doze tipos de climas, de acordo com a classificação climática de Köppen. O Estado de Mato Grosso pode ser incluso na zona A, clima tropical, fazendo parte de 81,4\% do território brasileiro com essa classificação (ALVARES et al., 2013). O clima de Rondonópolis, MT, é caracterizado, conforme a classificação climática de Köppen, como clima úmido, sub-úmido, megatérmico, com moderada deficiência hídrica no inverno e uma concentração de 30,6\% de evapotranspiração potencial atual, no trimestre mais quente: setembro, outubro e novembro (C2 A' S a') (INMET, 2015).

\subsection{Natureza e fonte de dados}

A pesquisa utilizou um estudo ecológico, de série temporal e analítico, de abordagem quantitativa e descritiva. Os dados foram de fonte secundária, relativos às séries de dados climáticos obtidas no Instituto Nacional de Meteorologia (INMET) e do número de casos de dengue notificados no Sistema de Informação de Agravos de Notificação (SINAN) (códigos A90-A91 da CID-10), do período de tempo estudado. Foram excluídos os casos não preenchidos e/ou ignorados.

As variáveis referentes aos dados climáticos foram distribuídas no valor médio da precipitação pluviométrica, da temperatura e da umidade 
relativa do ar, durante os meses do ano, obtidas por meio da disponibilidade do Banco de Dados Meteorológicos para Ensino e Pesquisa (BDMEP) do INMET da estação 83410 - Rondonópolis, MT, latitude: 16045', longitude: 54056', altitude: 284 m e sua situação é operante.

\subsection{Análise estatística dos dados}

A fim de quantificar os efeitos que as variáveis climáticas (precipitação pluviométrica, temperatura e umidade relativa do ar) exercem nos casos de notificação por dengue em Rondonópolis, MT, foram ajustados os modelos de Poisson e o binomial negativo pertencente à classe dos Modelos Lineares Generalizados (MLG), adotando-se um nível de significância de $5 \%(p<0,05)$.

A análise descritiva dos dados, em termos das porcentagens das variáveis dependente (casos totais de dengue de 2001 a 2015) e independentes (variáveis climáticas), foi obtida pela medida de tendência central (média, mediana) e de dispersão (desvio padrão e percentis), além do coeficiente de variação (CV). Sendo assim, foi ajustado o modelo de regressão de Poisson por se tratar de dados de contagem; no entanto, sempre que existe sobredispersão, torna-se necessário recorrer a outras distribuições, nomeadamente à distribuição Binomial Negativa. O fenômeno de sobredispersão ocorre quando é esperada uma distribuição de Poisson para a resposta, porém a variância é maior do que a resposta média. Uma causa provável desse fenômeno é a heterogeneidade das unidades amostrais que pode ser devido à variabilidade interunidades observacionais.

O teste $t$ foi utilizado para verificar possíveis significâncias dos parâmetros envolvidos no modelo, apresentando-se também seus respectivos desvios padrão, teste $t$ e o correspondente $p$-valor para a variável dependente em relação à significância das variáveis independentes.

Para avaliar a qualidade do ajuste do modelo, realizou-se a análise dos resíduos através dos gráficos normais de probabilidade junto com o teste qui-quadrado para verificar a adequabilidade do ajuste do modelo aos dados. Por fim, as análises foram realizadas com o auxílio do software estatístico R (R CORE TEAM, 2017 ). 


\subsection{Considerações Éticas}

O presente estudo fundamenta-se em dados secundários, de acesso público, que não constrangem grupos de populações e/ou indivíduos na apresentação dos resultados encontrados, sendo assegurada a confidencialidade das informações levantadas. Sendo assim, foram respeitados os aspectos éticos de pesquisa com seres humanos, de acordo com a Resolução n. 466/2012 (BRASIL, 2012). Além disso, esta pesquisa faz parte da pesquisa matricial intitulada "Distribuição espacial de doenças de notificação compulsória em Mato Grosso", que foi submetida ao Comitê de Ética em Pesquisa do Hospital Júlio Muller, por meio da Plataforma Brasil, sendo aprovada com parecer 1.571.782 e CAAE 54226316.1.0000.554.

\section{RESULTADOS}

Os casos notificados por dengue em Rondonópolis, MT, foram distribuídos de acordo com cada mês nos quinze anos amostrados (2001 a 2015) sendo um total de 18.953 casos. Os anos com maior número de casos foram 2010 ( $n=4.153,21,93 \%), 2013$ ( $n=3.396,17,92 \%), 2009$ ( $n=2.856,15,06 \%)$. $\mathrm{O}$ ano em que houve menor índice foi 2001, com apenas 4 notificações (0,02\%) (Tabela 1).

No tocante à classificação do tipo de dengue, destaca-se, na presente pesquisa, a dengue clássica com 8.512 (44,92\%) casos notificados. A dengue com complicação apresentou 120 (0,64\%) e a febre hemorrágica 140 $(0,74 \%)$ notificações.

Em relação às variáveis climáticas, a temperatura do município de Rondonópolis, MT, no ano de 2003 apresentou a menor média anual $\left(23,79^{\circ} \mathrm{C}\right)$, e no de 2015 , a maior média $\left(25,82^{\circ} \mathrm{C}\right)$; a umidade relativa do ar variou em 2005, apresentou a menor média anual, (71,53\%) e em 2014 a maior média (83,50\%); e a precipitação pluviométrica, nos anos de 2001 (94,725 mm/ano) e 2006 (127,3 mm/ano), apresentou a menor e maior proporção respectivamente (Tabela 1). 
Tabela 1 - Casos de dengue notificados e as variáveis climáticas de Rondonópolis, MT, no período de 2001 a 2015

\begin{tabular}{cccccc}
\hline \multicolumn{2}{l}{ Casos Notificados de Dengue } & \multicolumn{4}{c}{ Variáveis Climáticas } \\
\hline Ano & $\mathbf{n}$ & $\mathbf{\%}$ & $\begin{array}{c}\text { Precipitação } \\
\text { média anual }\end{array}$ & Temperatura & $\begin{array}{c}\text { Umidade } \\
\text { relativa do ar }\end{array}$ \\
\hline 2001 & 4 & 0,02 & 94,7 & 24,62 & 76,25 \\
2002 & 609 & 3,22 & 101,2 & 25,78 & 73,27 \\
2003 & 596 & 3,15 & 128,5 & 23,79 & 74,58 \\
2004 & 47 & 0,24 & 116,3 & 25,09 & 73,23 \\
2005 & 241 & 1,29 & 103,8 & 25,36 & 71,53 \\
2006 & 1.763 & 9,30 & 127,3 & 24,75 & 74,58 \\
2007 & 309 & 1,63 & 106,4 & 25,62 & 76,02 \\
2008 & 252 & 1,32 & 127,2 & 25,14 & 79,79 \\
2009 & 2.856 & 15,06 & 120,2 & 25,22 & 82,99 \\
2010 & 4.153 & 21,93 & 106,9 & 25,46 & 76,78 \\
2011 & 347 & 1,83 & 95,6 & 25,52 & 78,38 \\
2012 & 1.583 & 8,35 & 126,2 & 25,66 & 81,05 \\
2013 & 3.396 & 17,92 & 108,3 & 25,35 & 80,70 \\
2014 & 703 & 3,70 & 95,8 & 25,38 & 83,50 \\
2015 & 2.094 & 11,04 & 98,5 & 25,82 & 82,71 \\
\hline
\end{tabular}

Fonte: Dados do Sistema de Informação de Agravos de Notificação (SINAN e do Instituto Nacional de Meteorologia [INMET]).

Os meses de janeiro ( $n=3.977,20,99 \%)$, fevereiro ( $n=3.605,19,02 \%)$ e março $(n=2.713,14,31 \%)$ representam aqueles com maior quantidade de casos notificados nesse período. Em relação às variáveis climáticas, a média mensal da temperatura do município de Rondonópolis, MT, foi maior nos meses setembro $\left(26,8^{\circ} \mathrm{C}\right)$, outubro $\left(26,95^{\circ} \mathrm{C}\right)$ e novembro $\left(26,25^{\circ} \mathrm{C}\right)$, e menor, no mês de julho $\left(22,95^{\circ} \mathrm{C}\right)$. A média da menor, em agosto $(55,28 \%)$, considerado o mês mais seco. A precipitação pluviométrica apresentou umidade relativa do ar maior nos meses janeiro (88,71\%), fevereiro (88,32\%) e março (88,08\%), e a menor média, em agosto (4,2 mm/ano) e maior em janeiro (278,3 mm/ano) e dezembro (225,8 mm/ano); fato que pode corroborar para o estabelecimento de possíveis criadouros do mosquito Aedes Aegypti, favorecendo assim a sua 
proliferação e consequente aumento dos casos notificados (Tabela 2).

Tabela 2 - Número de casos de dengue notificados por mês e média mensal de precipitação, temperatura e umidade relativa do ar entre em Rondonópolis, MT, entre 2001 e 2015

\begin{tabular}{cccccc}
\hline Mês & Casos N & $\%$ & $\begin{array}{c}\text { Média } \\
\text { precipitação }\end{array}$ & $\begin{array}{c}\text { Média } \\
\text { temperatura }\end{array}$ & $\begin{array}{c}\text { Média umidade } \\
\text { relativa do ar }\end{array}$ \\
\hline Janeiro & 3.977 & $21 \%$ & 278,3 & 25,60 & 88,71 \\
Fevereiro & 3.605 & $19 \%$ & 118,6 & 25,66 & 88,32 \\
Março & 2.713 & $14 \%$ & 162,5 & 25,67 & 88,08 \\
Abril & 1.823 & $10 \%$ & 94,1 & 25,51 & 85,18 \\
Maio & 1.943 & $10 \%$ & 33,3 & 23,79 & 82,17 \\
Junho & 1.185 & $6 \%$ & 15,8 & 22,99 & 76,31 \\
Julho & 602 & $3 \%$ & 9 & 22,95 & 68,44 \\
Agosto & 385 & $2 \%$ & 4,2 & 24,65 & 55,28 \\
Setembro & 290 & $2 \%$ & 32,1 & 26,88 & 58,77 \\
Outubro & 372 & $2 \%$ & 115,1 & 26,95 & 71,89 \\
Novembro & 623 & $3 \%$ & 166,8 & 26,25 & 81,75 \\
Dezembro & 1.435 & $8 \%$ & 225,8 & 26,08 & 87,38 \\
\hline
\end{tabular}

Fonte: Dados do Sistema de Informação de Agravos de Notificação (SINAN e do Instituto Nacional de Meteorologia [INMET]).

Pode-se observar na Tabela 3 as principais estatísticas descritivas referentes às variáveis dependentes e independentes, destacando-se a discrepância entre os valores da média e variância, valores estes que deveriam possuir valor igual ou próximos para poder se adequar aos pressupostos do modelo de Poisson. Assim, os resultados apresentados indicam uma provável superdispersão dos casos de dengue no município em estudo, ou seja, variância superior à média. Quanto às medidas de forma da distribuição dos dados, os valores da assimetria e da curtose sugerem que os dados são bem dispersos em torno do seu valor médio. 
Tabela 3 - Distribuição estatística das variáveis dependentes e independentes e teste de normalidade de Anderson-Darling (AD) e correlação de Kendall (K) da variável dengue com relação às variáveis climáticas entre 2001 e 2015

\begin{tabular}{lrrrrrr}
\hline Estatísticas & Dengue & $\begin{array}{c}\text { Precipi- } \\
\text { tação }\end{array}$ & $\begin{array}{r}\text { Tempera- } \\
\text { tura Média }\end{array}$ & Umidade & $\begin{array}{c}\text { Temperatu- } \\
\text { ra Máxima }\end{array}$ & $\begin{array}{c}\text { Temperatu- } \\
\text { ra Mínima }\end{array}$ \\
Mínimo & 0,00 & 0,00 & 21,64 & 42,00 & 29,29 & 14,18 \\
Máximo & 1870,00 & 572,20 & 28,74 & 95,29 & 38,80 & 23,42 \\
1. Quartile & 7,00 & 16,93 & 24,29 & 69,53 & 32,04 & 17,81 \\
3. Quartile & 107,00 & 181,15 & 26,33 & 87,64 & 32,96 & 22,25 \\
Média & 112,79 & 111,59 & 25,29 & 77,79 & 33,13 & 20,16 \\
Mediana & 30,00 & 89,60 & 25,71 & 82,02 & 32,77 & 21,39 \\
Variância & 54572,74 & 11951,56 & 2,62 & 157,94 & 2,81 & 7,07 \\
Desvio Padrão & 233,61 & 109,32 & 1,62 & 12,57 & 1,68 & 2,66 \\
Assimetria & 4,24 & 0,99 & $-0,49$ & $-0,82$ & 0,74 & $-0,73$ \\
Curtose & 22,55 & 0,70 & $-0,46$ & $-0,36$ & 0,39 & $-0,94$ \\
Estatística AD & 27,47 & 5,89 & 3,35 & 5,23 & 2,66 & 9,57 \\
p-valor & $<0,001$ & $<0,001$ & $<0,001$ & $<0,001$ & $<0,001$ & $<0,001$ \\
Estatística K & --- & 3,8471 & $-0,406$ & 8,1790 & $-2,3984$ & 3,2217 \\
Rho & --- & 0,20 & $-0,02$ & 0,43 & $-0,13$ & 0,17 \\
p-valor & --- & $<0,001$ & 0,685 & $<0,001$ & 0,012 & $<0,001$ \\
\hline
\end{tabular}

Fonte: Elaborada pelos autores.

Foi realizado o teste de normalidade de Anderson-Darling (AD) para verificar a normalidade das variáveis em estudo. Sendo assim, de acordo com os resultados apresentados na Tabela 3, pode-se observar a não normalidade das variáveis analisadas ( $p$-valor $<0,001$ ), indicando que a correlação de Pearson não é adequada para se aplicar aos dados. Para verificar a correlação entre as variáveis (notificação dos casos de dengue e variáveis climáticas), foi utilizada a correlação de Kendall.

Por se tratar de dados de contagem, inicialmente ajustou-se a distribuição de Poisson. No entanto o ajuste do modelo forneceu deviance residual de $D(\mathbf{y} ; \mu)=22,395$ para 162 graus de liberdade, indicando fortes indícios de sobredispersão e evidência significativa de que o ajuste não seja adequado ( $p<0,001)$, o que é confirmado pelo gráfico normal de probabilidade apresentado na Figura 1. Tem-se, então, um modelo binomial negativo em que DENGUEi BN $(\mu \mathrm{i}, \phi)$. O gráfico normal de probabilidades bem como a deviance residual $D(\mathbf{y} ; \mu)=174,87$ para 149 graus de liberdade, fornecem 
indícios de ajustes adequados ( $p$-valor =0,051). A estimativa do parâmetro de dispersão foi de $\widehat{\phi}=1,04$.

Figura 1 - Gráficos normais de probabilidades referentes aos modelos loglinear de Poisson (a) e log-linear binomial negativo (b) ajustados aos dados de dengue, no período de 2001 a 2015 em Rondonópolis, MT

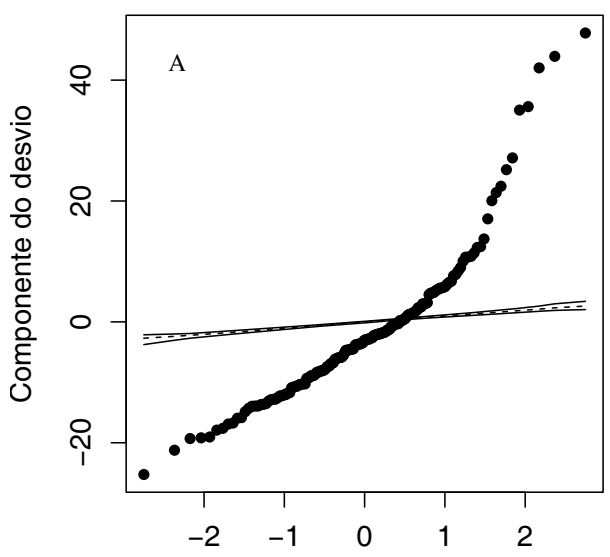

Percentil da $\mathrm{N}(0,1)$

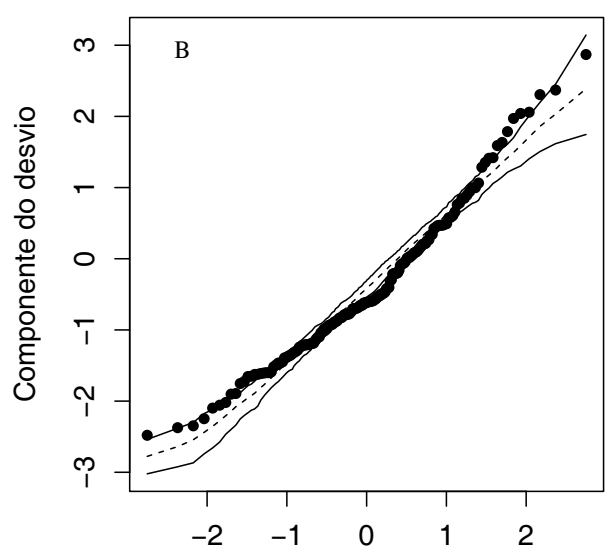

Percentil da $\mathrm{N}(0,1)$

Fonte: Elaborada pelos autores.

Os resultados apresentados na Tabela 4 representam as estimativas dos parâmetros do modelo e seus respectivos desvios padrão, teste $t$ e o correspondente $p$-valor para ocorrência de dengue, indicam que as variáveis climáticas temperaturas média e máxima do ar e umidade relativa do ar, foram significativas ao nível de $5 \%$ de probabilidade, no que se refere à explicação da taxa de aumento/decréscimo nos casos de dengue em Rondonópolis, MT. Nesta pesquisa, não houve associação significativa entre os casos de dengue e a precipitação pluviométrica. 
Tabela 4 -Estimativas dos parâmetros do modelo e seus respectivos desvios padrão, teste t e o correspondente p-valor para ocorrência de infecção respiratória aguda (IRA), no período de 2001 a 2015 em Rondonópolis, MT

\begin{tabular}{lcccc}
\hline \multicolumn{1}{c}{ Coeficientes } & Estimativa & Erro padrão & Teste t & p-valor \\
\hline Intercepto $\left(\hat{\beta}_{0}\right)$ & $-18,23420$ & 3,15705 & $-5,77$ & $<0,001$ \\
Temperatura média do $\operatorname{ar}\left(\hat{\beta}_{1}\right)$ & $-0,63386$ & 0,09853 & $-6,433$ & $<0,001$ \\
Temperatura máxima do $\operatorname{ar}\left(\hat{\beta}_{2}\right)$ & 0,57480 & 0,12164 & 6,370 & $<0,001$ \\
Umidade relativa do $\operatorname{ar}(\hat{\beta} 3)$ & 0,15906 & 0,01314 & 12,103 & $<0,001$ \\
\hline
\end{tabular}

Fonte: Elaborada pelos autores.

O coeficiente $\hat{\beta}_{1}==-0,63386$ indica a redução dos casos em função do aumento da temperatura média do ar, ou seja, existe uma relação inversa entre as variáveis em análise. Logo, espera-se que, para os meses com maiores registros de temperatura média do ar, ocorram menores índices de notificação por dengue. Sendo assim, $\exp (-0,63386)=0,46946$, estima-se que o número médio de casos de dengue diminua em aproximadamente $43 \%$ a cada 1 grau centígrado de aumento da temperatura média do ar (Figura 2a).

Já o coeficiente relacionado com a temperatura máxima do $\operatorname{ar} \hat{\beta}_{2}=$ 0,77480, foi positivo, indicando o aumento dos casos de dengue em função do aumento dessa variável, ou seja, existe uma relação direta. Assim, espera-se que, para os meses com maiores registros da temperatura máxima do ar, sejam observados os maiores índices de casos. Isto é, tomando exp (0, $57480)=1,776775$, estima-se que o número médio de casos de dengue aumente em torno de $77 \%$ a cada unidade de aumento na temperatura máxima (Figura 2b). 
Figura 2 - Variabilidade dos casos de dengue em relação à temperatura média do ar (a temperatura máxima do ar (b) em Rondonópolis, MT, de 2001 a 2015
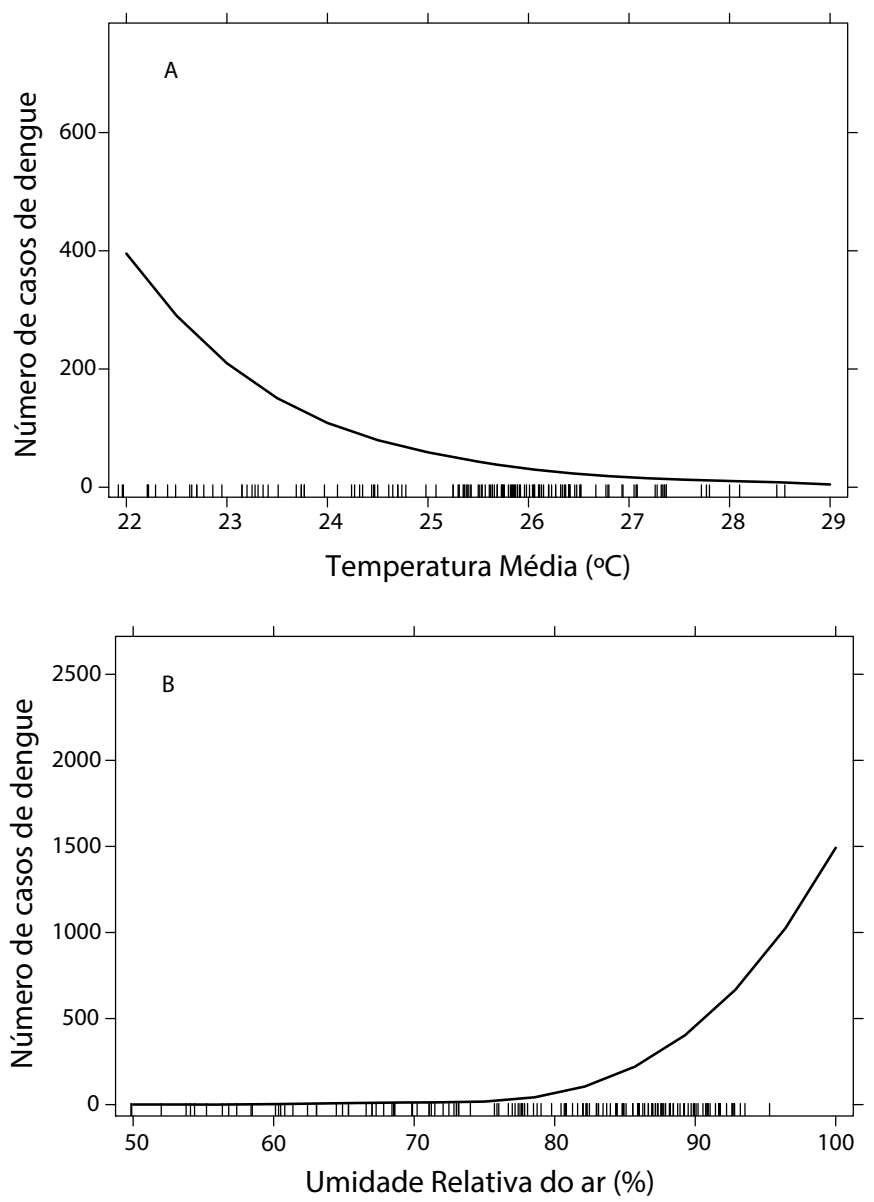

Fonte: Elaborada pelos autores.

O coeficiente relacionado com a umidade relativa do $\operatorname{ar}\left(\hat{\beta}_{3}=0,15906\right)$, também foi positivo, indicando o aumento dos casos de dengue em função do aumento dessa variável, ou seja, existe uma relação direta. Assim, espera-se que, para os meses com maiores registros da umidade relativa do ar, sejam observados os maiores índices de casos da doença. Isto é, tomando $\exp (0,15906)=1,172408$, estima-se que o número médio de casos de dengue aumente em torno de $17 \%$ a cada $1 \%$ de aumento acima da média da umidade relativa do ar (Figura 3). 
Figura 3 - Variabilidade dos casos de dengue em relação à umidade relativa do ar em Rondonópolis, MT, de 2001 a 2015

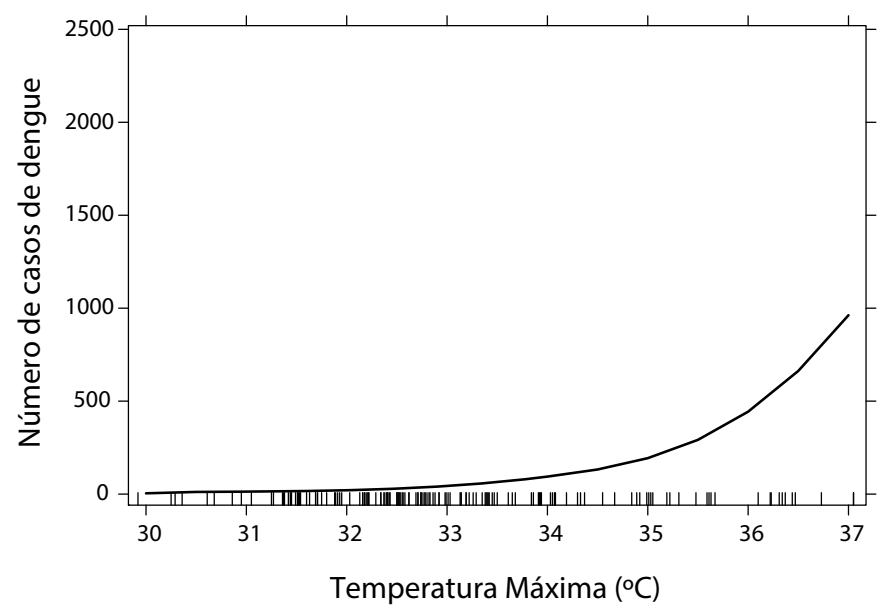

Fonte: Elaborada pelos autores.

\section{DISCUSSÃO}

No período analisado, houve predomínio dos casos de dengue nos anos 2010 ( $n=4.153,21,93 \%)$ e 2013 ( $n=3.396,17,92 \%)$. Em Barreiras, BA entre janeiro de 2007 e março de 2013, foram notificados 8.373 casos de dengue, dos quais $68,8 \%(n=5.757)$ eram casos prováveis da doença, sendo que houve registro de epidemia nos anos de 2009, 2011 e 2013 (COSTA; CALADO, 2016). No Brasil, ocorreram picos epidêmicos de dengue nos anos de 2002, 2008 e 2010, indicando que a transmissão da doença tem apresentado um comportamento cíclico, variando entre altas e baixas incidências (TEIXEIRA et al., 2013).

No município em estudo, houve subnotificação dos casos em 2001 $(0,02 \%)$, e a maioria dos casos $(53,7 \%)$ não foram classificados quanto ao tipo dengue. A subnotificação de casos, principalmente aqueles em que a assistência foi prestada pela rede privada, constitui-se em um dos problemas verificados no sistema, gerando informações limitadas para o planejamento e a execução das ações de prevenção e controle. A dengue faz parte da lista de doenças e agravos de notificação compulsória no Brasil; assim, todo 
caso suspeito ou confirmado deve ser notificado ao serviço de Vigilância Epidemiológica. Além disso, o serviço de combate às endemias deve ser informado para que atue no combate ao vetor nas regiões de maior incidência de dengue (PICINATO et al., 2015).

A maior parte dos casos de dengue nesta pesquisa foi notificada nos meses de janeiro, fevereiro e março. Casos semelhantes foram notificados nos meses de fevereiro, março e abril no município do Rio de Janeiro, RJ, no período de 2000 a 2013, sobressaindo com epidemias de dengue nos anos 2002, 2008, 2011, 2012 e 2013 (XAVIER et al., 2017). Situação diferente foi registrada em São Sebastião, SP, com 3.442 notificações de dengue, concentrando-se nos meses de abril a junho e com a estação do outono (RIBEIRO et al., 2006). Além disso, houve o aumento dos casos de dengue em Areia, PB, principalmente, nos meses de março, abril e maio e nas estações de verão e outono, com influência da temperatura e umidade do ar elevadas e dos altos índices pluviométricos no aumento desses casos (BARACHO et al., 2014).

Houve associação significativa entre as variáveis climáticas temperaturas média e máxima do ar e umidade relativa do ar e os casos de dengue em Rondonópolis, MT. Considera-se que janeiro e fevereiro foram os meses que, nesses anos de análise, apresentaram a maior prevalência de casos da doença nesse município, coincidindo com os meses em que foram registradas as maiores médias de umidade relativa do ar. Nos meses de setembro e outubro, registraram-se maiores médias mensais de temperaturas média e máximas do ar, concordando meses com números elevados de notificação de dengue. Nesses períodos, devem ser intensificadas as ações interdisciplinares para o controle dessas ocorrências de dengue no município.

As variáveis climáticas possuem relação estatística com os casos de dengue, demonstrando que o clima interfere na ocorrência da doença. Temperaturas mais baixas, níveis adequados de umidade e chuvas fornecem as condições para que o vetor possa se reproduzir e consequentemente transmitir a doença. Ainda se nota que a temperatura se relaciona ao ciclo do vetor, aumentando ou diminuindo a sobrevida do inseto adulto, impondo barreiras à distribuição geográfica da dengue (ANTONIO et al., 2015).

Esses dados relativos à associação dos casos de dengue e variáveis climáticas em Rondonópolis, MT, corroboram com o estudo de 
Pacheco et al. (2017), que destacaram a relação entre os casos de dengue e a temperatura do ar (média mensal) e umidade relativa do ar. Ainda no que tange à relação com a temperatura, em Cuiabá, MT, entre agosto de 2004 e agosto de 2005, uma das capitais mais quentes do país (média máxima por volta de $31^{\circ} \mathrm{C}$ ), foi evidenciada a correlação significativa com a temperatura máxima, média e mínima (MIYAZAKI et al., 2009). Em Teresina, PI, de 2002 a 2006, também observou-se correlação positiva forte entre incidência da dengue com a precipitação e a temperatura, particularmente no primeiro semestre de cada ano (MONTEIRO et al., 2009). No município do Rio de Janeiro, RJ, entre 2001 e 2009, o aumento de um grau na temperatura mínima em um mês gerou aumento de $45 \%$ no número de casos de dengue no mês seguinte (GOMES; NOBRE; CRUZ, 2012).

Não anuindo com os dados de Rondonópolis, MT, em Tangará da Serra, MT, no período de 2008 a 2010, a temperatura não variou consideravelmente, e esse fato pode não ser relevante para o desenvolvimento da população do mosquito transmissor da dengue. A temperatura ideal para o desenvolvimento desse agente etiológico varia de $24^{\circ} \mathrm{C}$ a $28^{\circ} \mathrm{C}$, o que demonstra que as altas temperaturas nem sempre estão relacionadas com a presença do vetor da dengue (FERNANDES et al., 2012). Resultados semelhantes foram encontrados em São Sebastião, SP, em que, entre 2001 e 2002, não foi observada associação entre os valores de temperatura média mensal, pluviosidade média mensal e número de casos de dengue (RIBEIRO et al., 2006).

Em consonância com o presente estudo, em Cascavel, PR, entre os anos de 2007 a 2001, um estudo identificou que, no período em que o número de casos apresentados foi maior, a umidade relativa do ar encontrava-se em um nível médio de $77 \%$ a $80 \%$. Já quanto ao menor número de casos, essa variável estava entre $67 \%$ e $72 \%$. Dessa forma, relaciona-se a maior ocorrência de dengue nos meses de janeiro, fevereiro e março, visto que a umidade relativa do ar em níveis superiores a 70\% favorece a proliferação do mosquito e o seu desenvolvimento (ANTONIO et al., 2015).

Em João Pessoa, PB, a dengue teve maiores incidências nos meses de verão, devido à temperatura e umidade elevadas, e, no outono, por decorrência das chuvas (SOUSA; DANTAS; LIMEIRA, 2007). Por outro lado, no município do Rio de Janeiro em 2005, não houve diferença estatística 
significante entre os períodos seco e chuvoso, sendo o efeito da sazonalidade descrito como baixo ou ausente na grande parte dos reservatórios analisados (MACIEL-DE-FREITAS et al., 2008).

Em Rondonópolis, MT, não houve associação significativa entre os casos de dengue e a precipitação pluviométrica. A relação entre os níveis dessa variável com os casos de dengue se modifica conforme o local de estudo. Em Boa Vista, RO, a correlação entre o número de casos notificados e as variáveis meteorológicas não foi encontrada, apresentando um padrão diferente a cada ano, destacando a existência de picos de casos tanto no período chuvoso quanto no seco (ROSA-FREITAS et al., 2003). Já em João Pessoa, PB, entre 2007 a 2011, houve maior quantidade de registros no período em que há maiores valores de precipitação, correspondendo a 85\% dos casos notificados (SILVA et al., 2015), assim como, entre 2007 e 2012, em Recife, $\mathrm{PE}$, foram confirmados 1.844 casos de dengue correlacionados a um acúmulo pluviométrico de 10215,02 mm (LIMA; MOREIRA; NÓBREGA, 2016).

Os índices pluviométricos também não foram significativos para a ocorrência de dengue no município de Rio de Janeiro, RJ entre os anos de 1986-2003. Considerou-se, como período de maior risco para o aumento do número de casos da doença, os verões quentes e secos, a temperatura mínima média acima de $22^{\circ} \mathrm{C}$, principalmente, do primeiro trimestre do período analisado e o volume de chuvas abaixo de $200 \mathrm{~mm} / \mathrm{mês}$ (CÂMARA et al., 2009).

Por outro lado, em 246 municípios no Estado de Goiás, entre janeiro de 2001 e dezembro de 2005, houve associação significativa dos picos da dengue com épocas de maiores volumes de pluviosidade média, que variam entre os primeiros quatro meses de cada ano (período de alta pluviosidade), e a redução desses casos entre junho e setembro (menor pluviosidade) (SOUZA; SILVA; SILVA, 2010). A temperatura do ar e a pluviosidade em Uberlândia, MG, influenciaram significativamente no aumento do número de criadouros e na dinâmica populacional do mosquito da dengue, sendo observado que $86,5 \%$ tornaram-se positivos no período chuvoso e somente 13,5\% no período seco (COSTA et al., 2008). 


\section{CONCLUSÃO}

Os resultados do presente estudo obtidos pela modelagem estatística sugerem uma maior associação entre o aumento da umidade e da temperatura máxima do ar e o aparecimento da dengue, em relação às demais variáveis independentes utilizadas no estudo. Além disso, revelaram elevados índices de notificação dos casos de dengue e, de forma geral, pode-se verificar que os maiores picos ocorrem nos meses quentes e úmidos, que corresponderam a janeiro, fevereiro e março.

A possibilidade de subnotificação de casos e da variabilidade da qualidade dos dados registrados no sistema de informação constitui uma limitação do estudo. Esses resultados sugerem que ações interdisciplinares de tomada de decisão nas políticas públicas de saúde e intersetoriais estejam voltadas prioritariamente para o verão, no intuito de controlar a doença no município.

Além disso, é necessário o aprofundamento dessas análises, considerando não somente da associação com os fatores ambientais climáticos, mas com os múltiplos fatores que envolvem os biológicos, geográficos, ecológicos, socioculturais e econômicos, os quais podem criar condições para o contato homem e vetor e colaborar com o aumento no número de notificações de dengue.

\section{REFERÊNCIAS}

ALVARES, Clayton Alcarde; STAPE, José Luiz; SENTELHAS, Paulo Cesar; de MORAES GONÇALVES, José Leonardo; SPAROVEK, Gerd. Köppen's climate classification map for Brazil. Meteorologische Zeitschrift, v. 22, n. 6, p. 71128, 2013.

ANTONIO, Geovane; SILVA, Claudinei Mesquita da; PEDER, Daiane Leyde de. Influência das alterações climáticas nos casos de dengue nos anos de 2007 a 2011 no município de Cascavel-PR. SaBios - Revista de Saúde e Biologia, Campo Mourão, v. 10, n. 1, p. 6-14, jan./abr. 2015.

BARACHO, Rosemery Cruz Monteiro; ISMAEL FILHO, Antônio; GONÇALVES, Amanda; NUNES, Sauma de Tássia Sousa; BORGES, Péricles de Farias. A influência climática na proliferação da dengue na cidade de Areia, Paraíba. Revista Gaia Scientia, João Pessoa, v. 8, n. 1, p. 65-73, 2014. 
BRASIL. Conselho Nacional de Saúde. Portaria n. 466/2012, de outubro de 2012. Dispõe sobre diretrizes e normas regulamentadoras de pesquisa com seres humanos. Diário Oficial da União, Brasília, DF, 13 de junho de 2013, Seção 1, p. 59 CÂMARA, Fernando Portela; GOMES, Adriana Fagundes; SANTOS, Gualberto Teixeira dos; CÂMARA, Daniel Cardoso Portela. Clima e epidemias de dengue no Estado do Rio de Janeiro. Revista da Sociedade Brasileira de Medicina Tropical, Uberaba, v. 42, n. 2, p. 137-40, mar./abr. 2009.

COSTA, Fernanda Silva; SILVA, Juliana Junqueira da; SOUZA, Carina Mara de; MENDES, Júlio. Dinâmica populacional de Aedes aegypti (L) em área urbana de alta incidência de dengue. Revista da Sociedade Brasileira de Medicina Tropical, Uberaba, v. 41, n. 3, p. 309-12, maio/jun. 2008.

COSTA, Isabelle Matos Pinheiro; CALADO, Daniela Cristina. Incidence of dengue cases (2007-2013) and seasonal distribution of mosquitoes (Diptera: Culicidae) (2012- 2013) in Barreiras, Bahia, Brazil. Epidemiologia e Serviços de Saúde, Brasília, v. 25, n. 4, p. 1-9, out./dez. 2016.

FERNANDES, Rosilainy Surubi; NEVES, Sandra Mara Alves da Silva; SOUZA, Cláudio Kléber Juiz de; GALVANIN, Edinéa Aparecida dos Santos; NEVES, Ronaldo José. Clima e casos de dengue em Tangará da Serra/MT. Hygeia, Uberlândia, v. 8, n. 15, p. 78-88, dez. 2012.

GOMES, Adriana Fagundes; NOBRE, Aline Araújo; CRUZ, Oswaldo Gonçalves. Temporal analysis of the relationship between dengue and meteorological variables in the city of Rio de Janeiro, Brazil, 2001-2009. Cadernos de Saúde Pública, v. 28, n. 11, p. 2189-97, nov. 2012.

INSTITUTO BRASILEIRO DE GEOGRAFIA E ESTATÍSTICA (IBGE). Cidades: Rondonópolis, Mato Grosso, 2017. Disponível em <http://cidades.ibge.gov.br/xtras/perfil. php?codmun=510760>. Acesso em: 23 jan. 2017.

INSTITUTO NACIONAL DE METEOROLOGIA (INMET). Precipitação Nacional de Meteorologia, 2015. Disponível: <http://www.inmet.gov.br/portal/> Acesso em: 23 jan. 2017.

LIMA, Rodolfo César; MOREIRA, Elvis Bergue Mariz; NÓBREGA, Ranyére Silva. A influência climática sobre a epidemia dengue na cidade do Recife por Sistema de Informações Geográficas. Revista Brasileira de Geografia Física, Recife, v. 9, n. 2, p. 384-98, 2016.

MACIEL-DE-FREITAS, Rafael; PERES, Roberto C; SOUZA-SANTOS, Reinaldo; LOURENÇO-DE-OLIVEIRA, Ricardo. Occurrence, productivity and spatial distribution of key-premises in two dengueendemic areas of Rio de Janeiro and their role in adult Aedes aegypti spatial infestation pattern. Tropical Medicine \& International Health, v. 13, n. 12, p. 1488-94, 2008. 
MIYAZAKI, Rosina Djunko; RIBEIRO, Ana Lúcia Maria; PIGNATTI, Marta Gisele; CAMPELO JÚNIOR, José Holanda; PIGNATI, Marina. Monitoring of Aedes aegypti mosquitoes (Linnaeus, 1762) (Diptera: Culicidae) by means of ovitraps at the Universidade Federal de Mato Grosso Campus, Cuiabá, State of Mato Grosso. Revista da Sociedade Brasileira de Medicina Tropical, Uberaba, v. 42, n. 4, p. 39297, jul./ago. 2009.

MONTEIRO, Eridan Soares Coutinho; COELHO, Mônica Elsy; CUNHA, lolanda Soares da; CAVALCANTE, Maria do Amparo Salmito; CARVALHO, Fernando Aécio de Amorim. Aspectos epidemiológicos e vetoriais da dengue na cidade de Teresina, Piauí - Brasil, 2002 a 2006. Epidemiologia e Serviços de Saúde, Brasília, v. 18, n. 4, p. 365-74, dez. 2009.

PICINATO, Mirelle Andréa de Carvalho; GRISOLIO, Ana Paula Rodomilli; CASELANI, Kelly; NUNES, Juliana Olivencia Ramalho; CARVALHO, Adolorata Aparecida Bianco; FERRAUDO, Antonio Sergio. Dengue: uma visão sobre o vetor urbano Aedes aegypti e a difícil interface do seu controle. Veterinária em Foco, Canoas, v. 13, n. 1, jul./ dez. 2015.

THE R Project for Statistical Computing. R: A language and environment for statistical computing. Vienna, Austria. 2017. Disponível em: <http://www.R-project.org/>.

RIBEIRO, Andressa F. Ribeiro; MARQUES, Gisela R. A. M.; VOLTOLINI, Júlio C.; CONDINO, Lúcia F. Associação entre incidência de dengue e variáveis climáticas. Revista de Saúde Pública, v. 40, n. 4, p. 671-6, 2006.

ROSA-FREITAS, Maria Goreti; TSOURIS, Pantelis; SIBAJEV, Alexander; WEIMANN, Ellem Tatiani de Souza; MARQUES, Alexandre Ubirajara; FERREIRA, Rodrigo Lopes; LUITGARDS-MOURA, José Francisco. Exploratory temporal and spatial distribution analysisof dengue notifications in Boa Vista, Roraima, Brazilian Amazon, 1999-2001. Dengue Bulletin, v. 27, p. 63-79, 2003.

SILVA, Alexandro Medeiros; SILVA, Richarde Marques da; ALMEIDA, Caio Américo Pereira de; CHAVES, José Jeferson da Silva. Modelagem geoestatística dos casos de dengue e da variação termopluviométrica em João Pessoa, Brasil. Sociedade \& Natureza, v. 27, n. 1, p.157-69, jan./abr. 2015.

SOUSA, Nadja Maria Nascimento; DANTAS, Renílson Targina; LIMEIRA, Rodrigo Cézar. Influência de variáveis meteorológicas sobre a incidência do dengue, meningite e pneumonia em João Pessoa-PB. Revista Brasileira de Meteorologia, v. 22, n. 2, p.183-92, 2007.

SOUZA, Sócrates Siqueira de; SILVA, Ionizete Garcia da; SILVA, Heloísa Helena Garcia da. Associação entre incidência de dengue, pluviosidade e densidade larvária de Aedes aegypti, no Estado de Goiás. Revista da Sociedade Brasileira de Medicina Tropical, Uberaba, v. 43, n. 2, p. 152-5, 2010. 
TEIXEIRA, Maria Glória; SIQUEIRA JÚNIOR, João Bosco; FERREIRA, Germano L C; BRICKS, Lúcia; JOINT, Graham. Epidemiological trends of dengue disease in Brazil (2000-2010): a systematic literature search and analysis. PLoS - Neglected Tropical Diseases, v. 7, n. 12, e2520, dez. 2013.

XAVIER, Diego Ricardo; MAGALHÃES, Mônica de Avelar Figueiredo Mafra; GRACIE, Renata; REIS, Izabel Cristina dos; MATOS, Vanderlei Pascoal de; BARCELLOS, Christovam. Spatial-temporal diffusion of dengue in the municipality of Rio de Janeiro, Brazil, 2000-2013. Cadernos de Saúde Pública, v. 33, n. 2, e00186615, 2017. 